\title{
Encapsulation of Nicotinamide into Cellulose Based Electrospun Fibers
}

\author{
Ahmed A Nada*, Ahmed G Hassabo, Amina L Mohamed, Saad Zaghloul \\ Textile division, Pre-treatment and Finishing of Cellulosic Fibers Department, National Research Center, Giza, Egypt.
}

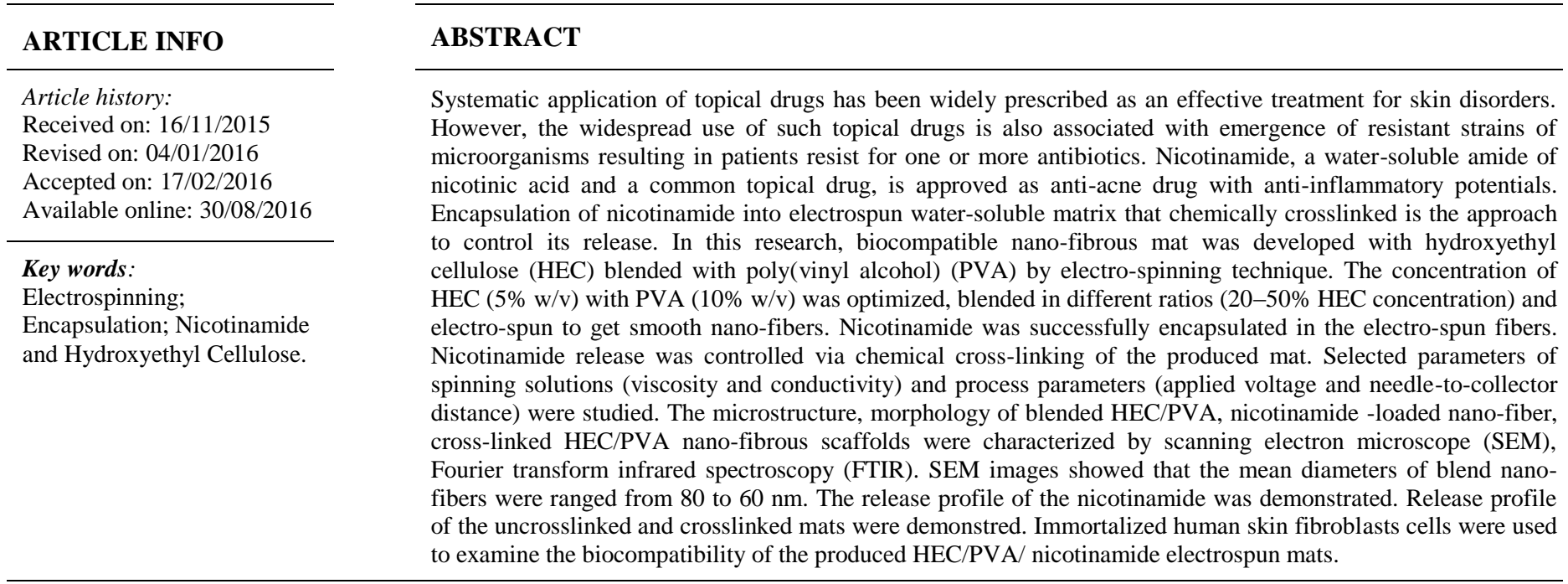

\section{INTRODUCTION}

Systematic application of topical drugs has been widely prescribed as an effective treatment for skin disorders. However, the widespread use of such topical drugs is also associated with emergence of resistant strains of microorganisms resulting in patients with resistance for one or more antibiotics (Leyden, 1976). As a result, new precautions were proposed to restrict the use of topical drugs as therapeutic treatments (Shalita et al., 1995). These observations led to new developments in drug delivery for topical applications to control the drug delivery. Controlling drug release provides sustained delivery over specific period of time and reduces the amount of drug necessary to cause the same effect. In order to control the release profile of topical

\footnotetext{
* Corresponding Author

Ahmed A Nada, Textile division, Pre-treatment and Finishing of Cellulosic Fibers Department, National Research Center, Giza, Egypt.

Email: aanada@ncsu.edu
}

drugs, different systems of drug encapsulation have been employed over the last few decades (Huang and Brazel, 2001) such as hydrogels (Cheng et al., 2014), films (Rezvanian et al., 2016), hydrocolloids (Jin et al., 2015), liposomes (Abdel-Azeem and Nada, 2015) and non-woven mats (Supaphol and Suwantong, 2012). Among those systems of encapsulation, electrospinning of the carbohydrate polymers has been established due to its simple procedure and its potentials (Ghosh, 2006). Electrospinning technique can produce different-sized fibers using electrostatic forces that result in the creation of fibers with diameters typically between 10 and 150 nanometers or more. Such fibers have been produced in dry process (natural method) with limited pollution and benign environmental impact for various applications. In woundcare applications, electrospun fibers have been produced whether from medicated polymers (Lee et al, 2009; Nada et al., 2014) or from polymers incorporated with active agents (Charernsriwilaiwat et al., 2013; Taepaiboon et al., 2007). 
However, solubility of such active agents with carrier polymers in common solvent was and still the main problem. On the other hand, water-soluble drugs such as nicotinamide when electrospun into water-soluble polymers that can dissolve in blood or tissue fluids in the wound bed resulting sort of medical complications (Xu et al., 2005). Therefore, crosslinking of the electrospun mats loaded with drugs has become necessary to control polymer dissolution and drug release (Aravamudhan et al., 2014). In this work, nicotinamide was loaded into electrospun fibers fabricated by the electro-spinning of hydroxyethyl cellulose/Poly-(vinyl alcohol). Citric acid was used as a corsslinker to control nicotinamide release. The microstructures of the obtained nanofibers were analyzed by scanning electron microscopy (SEM). Fourier transform infrared spectroscopy (FT-IR) was used to prove the chemical modifications. Electrospinning parameters were studied to obtain clear, smooth and bead-free fibers in different diameter size. The cytotoxicity test was considered to examine the final product biocompatibility.

\section{EXPERIMENTAL}

\section{Materials}

Hydroxyethyl cellulose, medium viscosity $(75-125 \mathrm{cP}$ at $2 \%$ in water, $20^{\circ} \mathrm{C}$ ), was purchased from Fluka. Poly(vinyl alcohol) (88\% hydrolyzed with molecular weight 25,000) was purchased from Sigma-Aldrich. Nicotinamide, sodium hydroxide, citric acid and sodium hypophosphite (sodium salt of phosphorous acid) was purchased from Fisher. MTT dye was purchased from Sigma-Aldrich. All reagents are laboratory grade chemicals.

\section{Methods}

\section{Preparation of electro-spinning solution}

The hydroxyethyl cellulose solution with concentration 5 $\%$ w/v was prepared by dissolving $5 \mathrm{~g}$ of hydroxylethyl cellulose powder in $100 \mathrm{ml}$ distilled water for $4 \mathrm{~h}$ at room temperature until a clear solution was obtained with a slight increase in viscosity. Polyvinyl alcohol solution of $10 \% \mathrm{w} / \mathrm{v}$ was prepared by dissolving $10 \mathrm{~g}$ of PVA granules in $100 \mathrm{ml}$ distilled water with moderate stirring at $60^{\circ} \mathrm{C}$ for $24 \mathrm{~h}$. To ensure a complete dissolution and homogeneous solution, both solutions were stirred continuously for $12 \mathrm{~h}$ at $60^{\circ} \mathrm{C}$.

Hydroxyethyl cellulose was then blended in polyvinyl alcohol solution with 3 different weight ratios of HEC: PVA namely; 50: 50, 40: 60 and 30: 70 and stirred overnight to get homogeneous mixture for electro-spinning. The nicotinamide drug was added with 3 different concentrations for the electrospun HEC: PVA ratio.

\section{Electro-spinning process}

The electrospinning apparatus was designed as shown in Figure (1) and described on previous work (Nada et al., 2014). A typical electrospinning device has three main components namely; power supply, polymer solution delivery system and collector. Briefly, a programmable syringe pump was used to deliver polymer solutions at controlled flow rates. The polymer solution was given an electric potential which exceeds its forces of surface tension through the high voltage $(0-30 \mathrm{kV})$ power supply.

Consequently, a fine polymer fiber was ejected from the syringe needle (18 gauge, $1.27 \mathrm{~mm}$ ) towards the collector performing ultra thin fibers deposited on an aluminium foil sheet of dimension $10 \mathrm{~cm} \mathrm{X} 10 \mathrm{~cm}$ (Hurst, 2011). The aluminium foil sheet was placed in the center of a piece of wood $(15 \mathrm{~cm} \mathrm{X} 15 \mathrm{~cm})$ which attached to a copper cylinder connected to the grounded cable. The polymeric solution was filled into a plastic syringe that has stainless steel blunt needle tip and connected to the DC voltage source.

The height of the needle tip was adjusted to face and aligned with the center of the vertically oriented aluminium foil. While, distance between the needle tip and collector was changed from $5 \mathrm{~cm}$ to $25 \mathrm{~cm}$ which refers to the air-gap distance. All experiments were carried out in ventilated chamber made of wood at room temperature.

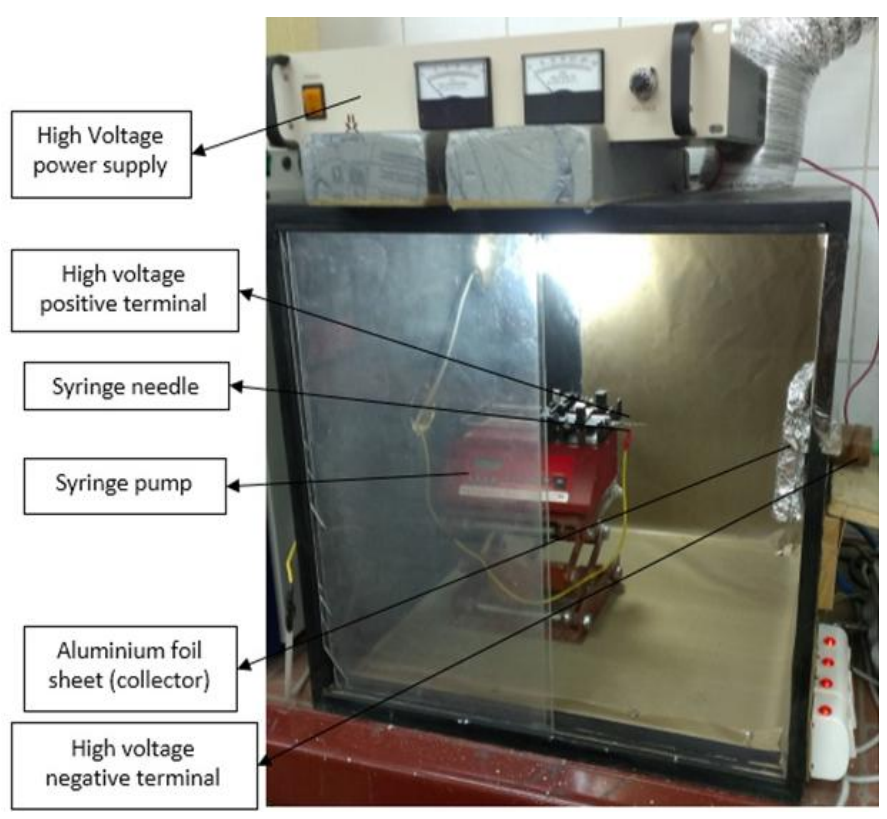

Fig. 1: Electro-spinning apparatus.

\section{Testes and analysis}

\section{Fourier Transform Infra-Red Spectroscopy (FT-IR)}

FTIR instrument model 460 plus Jasco, (Micro Analytical Center, Cairo Univeristy, Egypt) was used to analyse the spectrum of samples. Samples were mixed and pressed with $\mathrm{KBr}$ powder to form $\mathrm{KBr}$ pellets subjection to high pressure using a manual pellet maker. Using DTGS (Globar) laser source to provide different wavenumbers $(v)$ through the intrferometer to produce a $\left(4000-400 \mathrm{~cm}^{-1}\right)$ wavenumber range. Software was setted up to scan the background and samples at certain number of scans (64), certain resolution (4). A proper time should be taken (ca. $15 \mathrm{~min}$ ) to attain a clear spectrum out of moisture and carbon dioxide (Skoog et al., 2007a). 


\section{Scanning electron microscopy (SEM)}

The electro-spun fibers morphology was assessed using scan electron microscopy (Quanta 250 FEG (Field emission Gun), located at the national research center (NRC). Samples (electrospinning mats), deposited on aluminium sheet, fixed to sample holder, were coated with a layer of gold (100 $\AA$ ) in vacuum using sputter coater S150A Edwards-England to produce conductive surface. Gold- coated mats were placed in the microscope champer. Features of sample morphology were obtained in 5-10 kV (Verreck et al., 2003). Fiber diameters were calculated from the SEM micrographs using ImageJ software.

\section{Viscosity and conductivity}

The viscosity of the polymer solutions was determined by a rotation viscometer (Brookfield) at room temperature. The electrical conductivity of polymer solutions prepared for electrospinning was measured using Myron L Ultrameter II, Model 6P.

\section{In vitro drug release experiment}

Releasing profile of the encapsulated drug from electrospun mat was determined as follow: $50 \mathrm{mg}$ of electro-spun mat loaded by $2 \% \mathrm{w} / \mathrm{w}(1 \mathrm{mg})$ nicotinamide was incubated in $10 \mathrm{~mL}$ releasing medium (phosphate buffer solution (PBS), $\mathrm{pH} 7.4$ at $37^{\circ} \mathrm{C}$ ) in shaking incubator for 24 hours. At different time points, 1 $\mathrm{ml}$ of the released medium was taken out using syringe filter $(0.2$ $\mu \mathrm{m}$ membrane). Then, $1 \mathrm{ml}$ of fresh medium was replaced to keep the total amount constant. The concentration of the released amount of drug was calculated by measuring the absorbance readings at $289 \mathrm{~nm}$ using Shimadzu UV-visible recording spectrophotometer model-UV 240 (NRC, Egypt). The released amount was calculated from standard calibration curve (Nada et al., 2011)

\section{Encapsulation efficiency}

The encapsulation efficiency (EE) was determined of the remaining amount of the nicotinamide encapsulated in PVA/HEC electrospun fibers compared to the starting amount:

$$
\mathrm{EE} \%=\frac{\text { Practical Drug loading }}{\text { Theoretical Drug loading }} \times 100
$$

The theoretical nicotinamide was calculated as $2 \%(\mathrm{w} / \mathrm{w})$ of 50 $\mathrm{mg}$ of the electrospun mat $=1 \mathrm{mg}$. Standard solutions were prepared for the calibration curve to cover the range of drug used. The practical loading was measured after $48 \mathrm{hrs}$ incubation in 10 $\mathrm{mL}$ in $\mathrm{PBS} \mathrm{pH} 7.4$ at $37^{\circ} \mathrm{C} .1 \mathrm{ml}$ of the released medium was taken out using syringe filter $(0.2 \mu \mathrm{m}$ membrane $)$. The concentration of the released amount of drug was calculated by measuring the absorbance readings at $289 \mathrm{~nm}$ using Shimadzu UV-visible recording spectrophotometer model-UV 240 (NRC, Egypt). The released amount was calculated from standard calibration curve.

\section{Cytotoxicity assessment}

The cytotoxicity test was conducted in adaption from
ISO 10993-5 standard test method in which human immortalized skin fibroblasts (hTERT-BJ1) were used for all studies. hTERTBJ1 was kindly provided from Prof. Stig Linder, Karolinska Institute, Sweden. The choice of fibroblast cell line is based on their role on producing the protein associated in extracellular matrix (ECM) synthesis and their crucial role in wound healing process.

In typical procedure, cells were maintained in Dulbecco's modified eagle medium (DMEM):F12 Medium (nutrient mixture) $/ 10 \%$ (w/v) fetal bovine serum (FBS) and were incubated at $37^{\circ} \mathrm{C}$ in $5 \% \mathrm{CO}_{2}$ and $95 \%$ humidity. Cells were seeded into wells in 96-well plate and at a density of 30000 cells per well. After incubation for $48 \mathrm{hrs}$ the culture medium were replaced by extracted media of different concentrations $(12.5,25,50$ and 100 ppm $\{\mu \mathrm{g} / \mathrm{mL}\}$ ) of the tested compounds (each sample was tested in three different wells).

Pure HEC, pure PVA, nicotinamide and 2\% (w/v) loaded drug into crosslinked electrospun mat are the tested compounds for this test. Samples were sterilized under ultraviolet (UV) light for $20 \mathrm{~min}$ in a laminar flow before extraction. Cell culture medium, without additional reagents, was used as control. The plate was incubated again for $24 \mathrm{hrs}$. The amount of living cells was determined by the MTT assay.

The culture medium was aspirated and replaced by $40 \mathrm{uL}$ of MTT solution $(2.5 \mathrm{mg} / \mathrm{mL})$. Then, the solution was incubated for another $4 \mathrm{hr}$ at $37^{\circ} \mathrm{C}$. The solution was aspirated and $200 \mu \mathrm{l}$ $10 \%$ sodium dodecyl sulphate (SDS) in deionized water was added to each well to dissolve the formazan crystals and incubated overnight at $37^{\circ} \mathrm{C}$. The absorbance was measured using a ChroMate microplate (Awareness Technology, U.S.A.) reader at $595 \mathrm{~nm}$ and a reference wavelength of $690 \mathrm{~nm}$ (Nada et al., 2015).

\section{Differential Scanning Calorimetry (DSC) analysis}

Differential Scanning Calorimetry (DSC), Model Perkin

Elmer, equipped with a refrigerated cooling system and nitrogen as a purging gas, was used to determine the glass transition temperature $(\mathrm{Tg})$. About $4 \mathrm{mg}$ of dry sample were weighted into standard DSC hermetic aluminum sample pans. The analysis was performed in temperature ramp from $25^{\circ} \mathrm{C}$ to $250^{\circ} \mathrm{C}$ at rate of $10^{\circ} \mathrm{C} /$ minute (Skoog et al., 2007b)

\section{Thermogravimetric analysis (TGA)}

Thermogravimetric (TGA) analysis were performed with a Mettler-Toledo DTA/TGA instrument in the temperature range from 50 to $500^{\circ} \mathrm{C}$, at a rate of $10^{\circ} \mathrm{C} / \mathrm{min}$, under nitrogen flow (Hassabo et al., 2015; Hassabo, 2014)

\section{Statstical analysis}

Results were expressed as a mean value with its standard deviation (mean \pm S.D.) of each sample that is repeated three times $(n=3)$. Statistical analysis was performed with student's t-test and differences were considered as significant at p-values under 0.05 . 


\section{RESULTS AND DISCUSSIONS}

\section{Fiber Formation}

Encapsulated nicotinamide into PVA/HEC mat was performed via studying the electrospinning parameters. Flow rate $(0.2 \mathrm{~mL} / \mathrm{h}), \mathrm{PVA} / \mathrm{HEC}(70: 30(\mathrm{v} / \mathrm{v}))$, nicotinamide concentrations $(2,4$ and $6 \% \mathrm{w} / \mathrm{w})$, voltage applied $(15 \mathrm{kV})$ and needle tip-toreceiver distances $(15 \mathrm{~cm})$ were be able to produce electrospun fibers. The production sequence of the PVA/HEC/Drug electrospun fibers is illustrated in Scheme 1

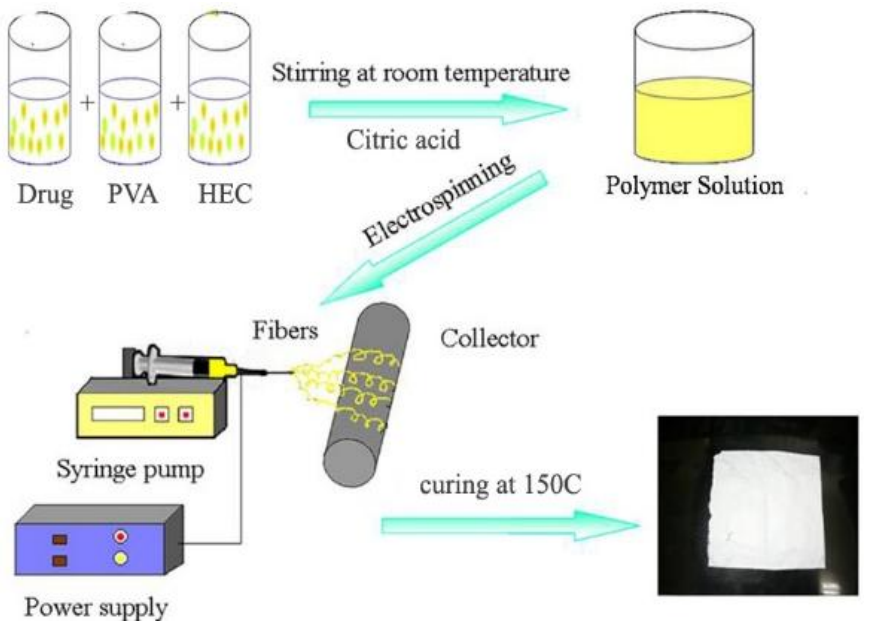

Scheme 1 Illustrating the production steps of electro-spun fibers.

The influence of polymers concentration, viscosity $(\eta)$ and conductivity $(\lambda)$, on the fiber formation and diameters is summarized in Table 1. In this research, the ratio of both polymers PVA and HEC are varied from 50/50 to 80/20.

HEC raito lower than $30 \%$ showed only sprayed beads. The latter phenomenon can be attributed to an increase in the hydrogen bonding between polymer chains which leads to higher surface tension to the spinning solution. Such high surface tension requires high voltage to perform the Taylor cone. Therefore, solution moves very fast to the target without enough time to stretch out in the gab distance to end up with beads instead of fibers.

Table 1 data showed that $100 \%$ HEC solution shows high electrical conductivity $(2300 \mu \mathrm{s})$ and by increasing the PVA in solutions electrical conductivity has decreased accordingly to 1380 us with $70 \%$ PVA (v/v). On other words, HEC ratio at $30 \%$ and PVA $70 \%(\mathrm{v} / \mathrm{v})$ leads to a successful fiber formation process providing bead-free electrospun fibers. This may be due to the decrement of the polymers mixture surface tension that requires lower voltage to perform the Taylor cone (Yang et al., 2004). The viscosity and electrical conductivity values of PVA/HEC solutions were listed in Table 1. As HEC ratio decreased (50 to $20 \%$ ), both viscosity and electrical conductivity are decreased from 1670 to $1290 \mu \mathrm{s}$ and 1504 to $710 \mathrm{cP}$. This phenomenon may be resulting from the decreasing of hydrogen bonding between polymers chains. Table 1 shows also insignificant changes in solutions viscosity and electrical conductivity with drug additions (from 940 to $948 \mathrm{cP}$ for 2 to $6 \%$ drug respectively). Such inconsiderable changes in both viscosity and conductivity have shown no impact on the electrospinning final results.

Table 1: Electrical conductivity ( $\mu \mathrm{s})$ and viscosity $(\mathrm{cP})$ of polymeric solutions.

\begin{tabular}{lll}
\hline & $(\mu \mathrm{s})^{*}$ & $(\mathrm{cP}) * *$ \\
\hline Deionized water & 1000 & 1 \\
PVA $(10 \%)$ & 583 & 1300 \\
HEC $(5 \%)$ & 2300 & 525 \\
PVA/HEC (50/50) & 1670 & 1504 \\
PVA/HEC (60/40) & 1490 & 1230 \\
PVA/HEC (70/30) & 1380 & 804 \\
PVA/HEC (80/20) & 1290 & 710 \\
PVA/HEC/Drug $(2 \% \mathrm{w} / \mathrm{w})$ & 1340 & 940 \\
PVA/HEC/Drug $(4 \% \mathrm{w} / \mathrm{w})$ & 1350 & 944 \\
PVA/HEC/Drug $(6 \% \mathrm{w} / \mathrm{w})$ & 1355 & 948 \\
\hline
\end{tabular}

** $\mathrm{cP}=$ centipoises $; \mu \mathrm{s} *=$ microsiemens

\section{Chemical crosslinking for HEC/PVA/nicotinamide mat}

Nicotinamide when electrospun into HEC/PVA, watersoluble, polymers that can dissolve in blood or tissue fluids in the wound bed resulting sort of medical complications (Xu et al., 2005). Therefore, chemical crosslinking of the electrospun mats loaded with drugs has become necessary to control polymer dissolution and drug release. Citric acid/sodium hypophosphite system (Reddy and Yang, 2010) was used as a crosslinker for the PVA/HEC nanofibers. Our approach involved the synthesis of HEC/PVA crosslinked via a bi-carboxylic acid compound namely; citric acid in the presence of acid catalyst (sodium hypophosphite) to produce a new composite can trap nicotinamide and control its release. In brief, $5 \%(\mathrm{w} / \mathrm{w})$ of citric acid and sodium hypophosphite $(50 \% \mathrm{w} / \mathrm{w}$, of citric acid used) were added to a mixture of 3 parts of HEC (5\% w/v) and 7 parts of PVA (10\% $\mathrm{w} / \mathrm{v})$. The mixture was electrospun and mat was air dried and then cured in hot air oven at $150^{\circ} \mathrm{C}$ for 3 minutes. Mat was thoroughly washed with distilled water to removed unbounded citric acid. The possible reaction mechanism is presented in Fig.2.

In this mechanism, acid catalyst (sodium hypophosphite) was used to increase the reactivity of the carbonyl carbon (A) as an electrophile by the protonation of the carbonyl oxygen to facilitate its attack by the alcohol oxygen (B) of either HEC or PVA. After the nucleophilic attack, one proton transferred to convert one of the $\mathrm{OH}$ groups into a good leaving group and finally $(\mathrm{C})$ eliminate a water molecule (D). Compound (F) represents the esterification reaction on one side of citric acid with R' as HEC and R" as PVA.

Figure 3 shows the FT-IR spectral data of the PVA/HEC/Drug electro-spun fibers with and without the crosslinker. It is clear that, the peak corresponding to carbonyl group observed at $1710 \mathrm{~cm}^{-1}$ which indicating that, the esterification reaction has taken place. In addition, the intensity of free hydroxyl group at $3250 \mathrm{~cm}^{-1}$ was decreased indicating the consumption of the hydroxyl groups through the esterification between the citric acid and polymers chains. Since the crosslinked samples washed thoroughly with water to remove unbounded citric acid, the recorded carbonyl group confirms the chemical reaction between HEC and PVA with citric acid. 

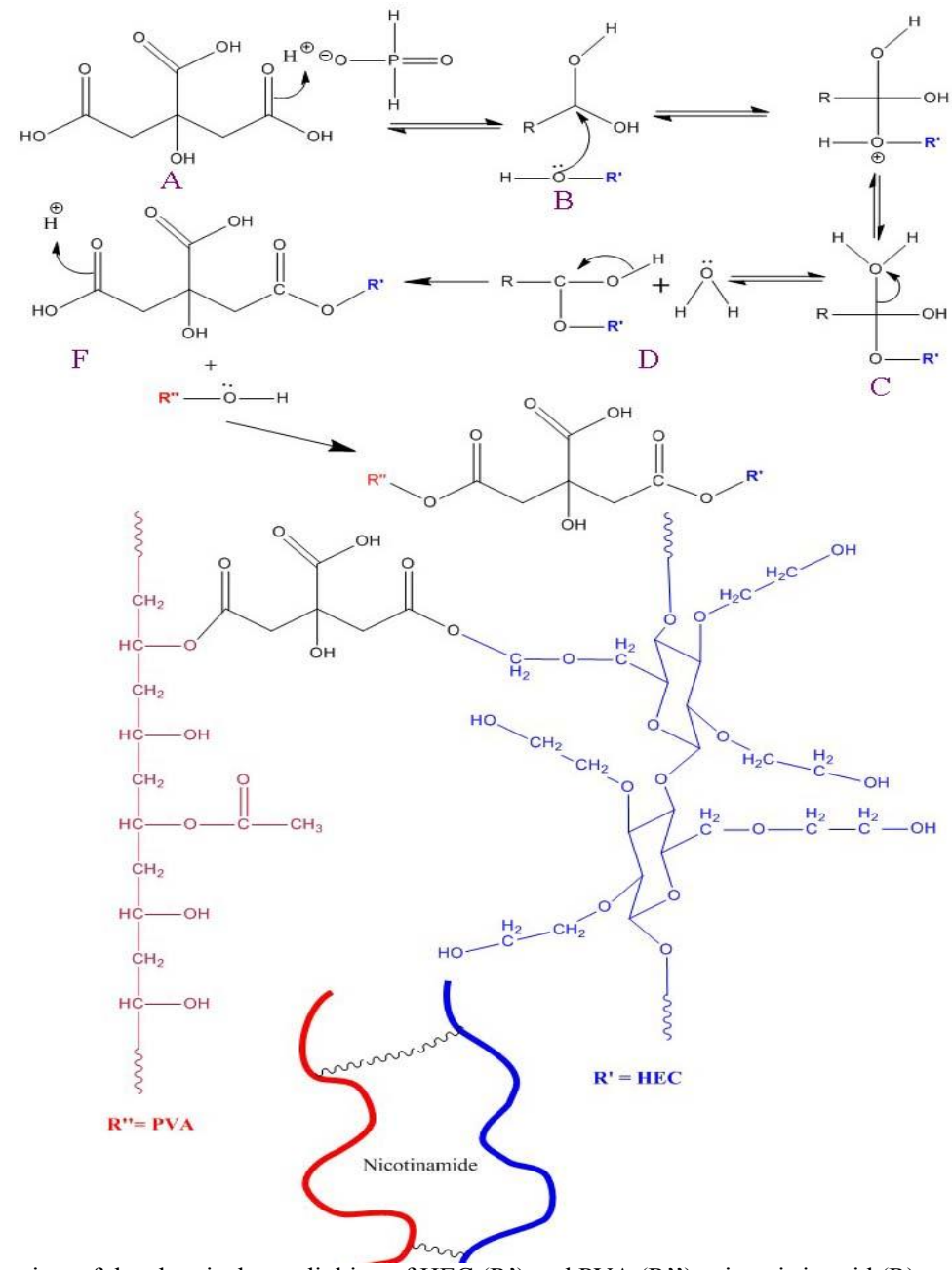

Fig. 2: Suggested mechanism of the chemical crosslinking of HEC (R') and PVA (R') using citric acid (R) and sodium hypophosphite.

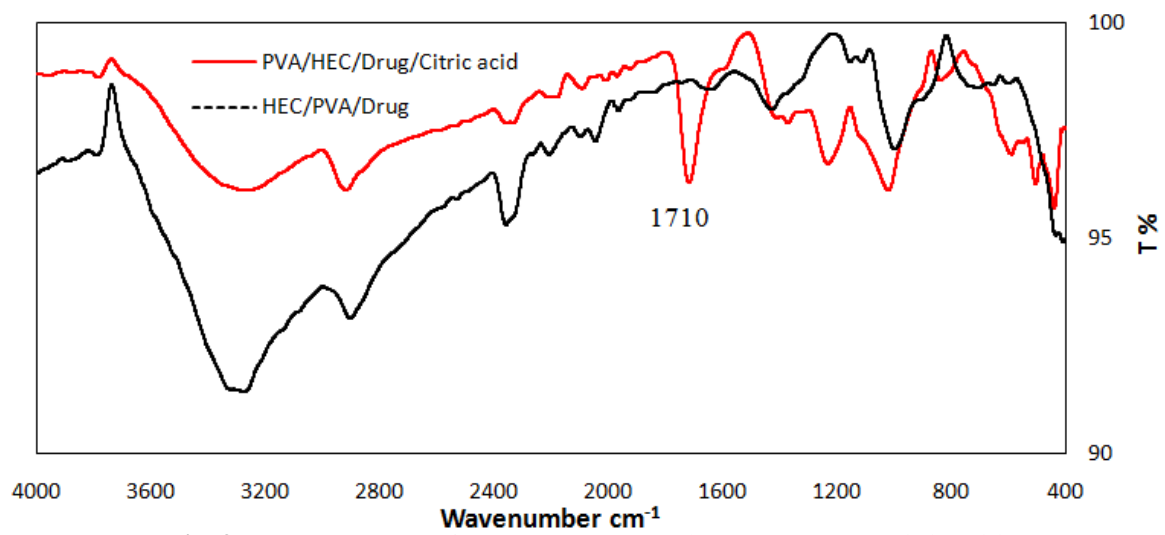

Fig. 3: FT-IR spectrum of PVA/HEC/Drug and PVA/HEC/Drug/citric acid.

\section{Morphology and Properties of Produced Fibers}

\section{Scanning electron microscope (SEM)}

Four difference ratios of polymer solutions were prepared and used for the electro-spinning system to form electrospun fibers (PVA: HEC; 50:50, 60:40, 70:30 and 80:20). Because of high viscosity and conductivity of both 50:50 and 60:40 polymer solutions, nothing was observed but spherical droplets. In addition, the other two formulations showed successful results in producing nanofibers. Fig. 4 shows SEM images for electrospun fibers in which both polymer ratios below (70: 30) PVA: HEC showed droplets (beads) throughout the whole sample, but polymer ratio (70: 30 and 80:20) PVA: HEC gives nanofibers. In addition, 70:30 ratio showed smooth fibers than 80:20 and 80:20 have some spots as beads on the surface. This is may be attributed to the lower viscosity and conductivity of PVA: HEC (80: 20) if compared to PVA: HEC (70: 30). Furthermore, adding the drug to the polymer 
formulations during electro-spinning process led to bead-free fibers with different in diameters. In case of adding $2 \%(\mathrm{w} / \mathrm{w})$ drug, the fiber morphology have a spherical and containing beads on the whole fiber surface, and the fiber looks homogenous with diameter range 60-80 $\mathrm{nm}$. Increasing the nicotinamide concentration led to some cracks appeared on overall fiber surface. At concentration to $6 \%(\mathrm{w} / \mathrm{w})$ of nicotinamide, the resulting nanofibers are containing some spindle-like beads along with the cracks.

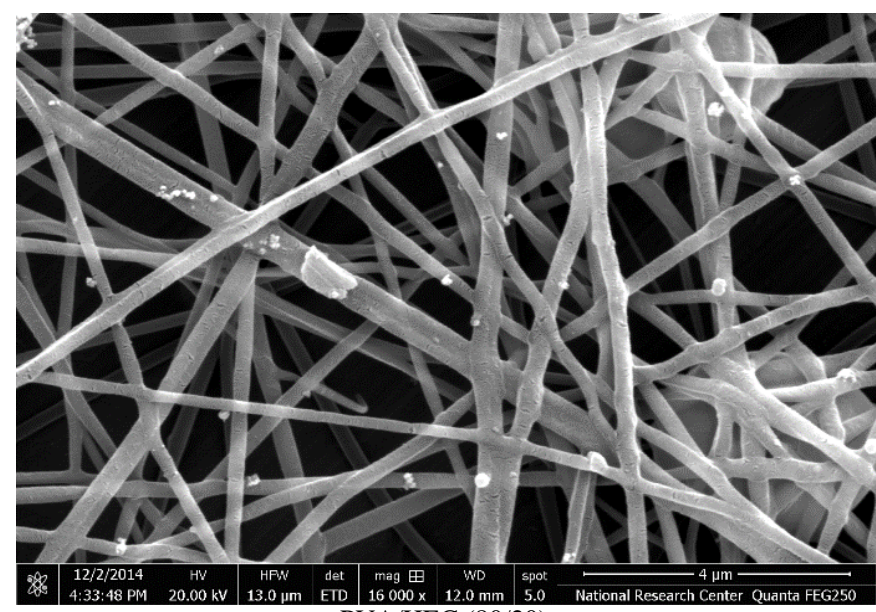

PVA/HEC (80/20)
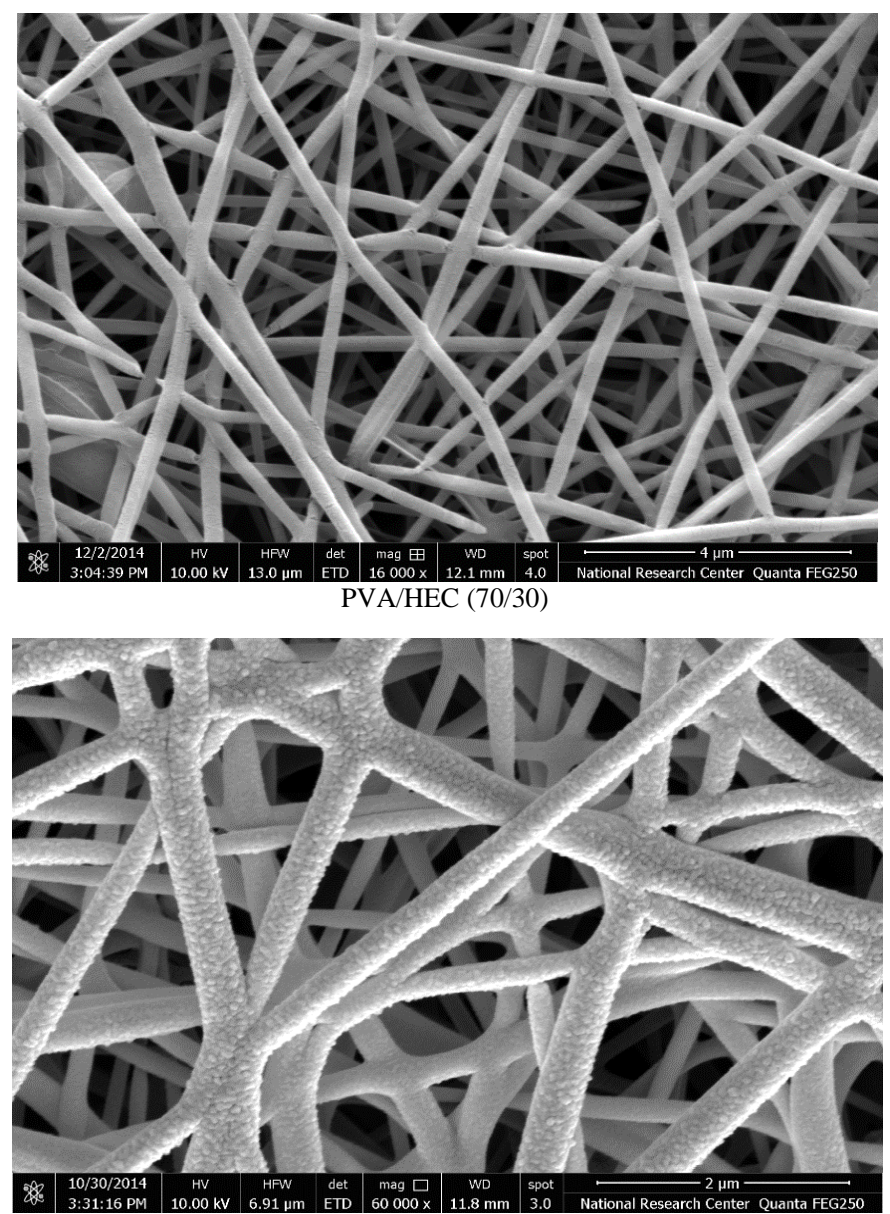

PVA/HEC loaded with $2 \%$ (w/w) Drug

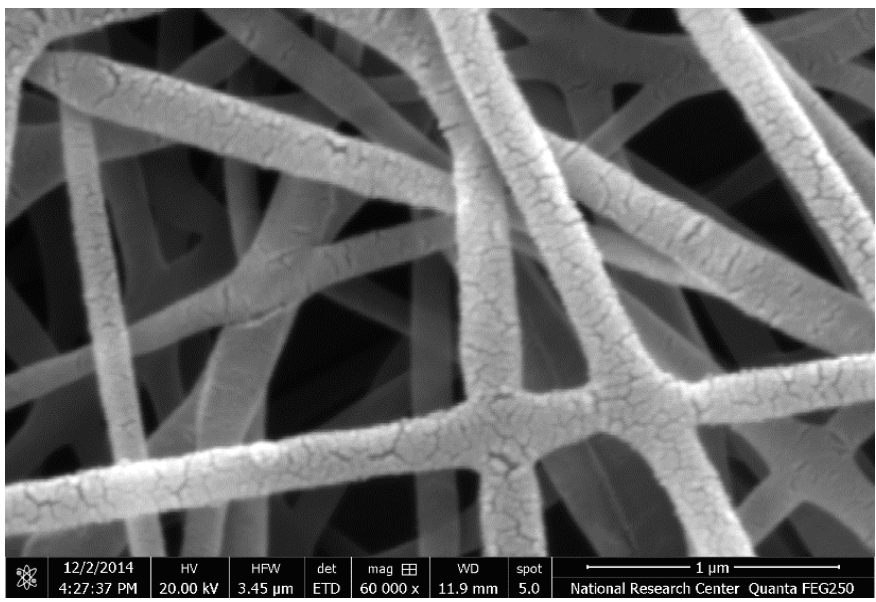

PVA/HEC loaded with 4\% (w/w) Drug

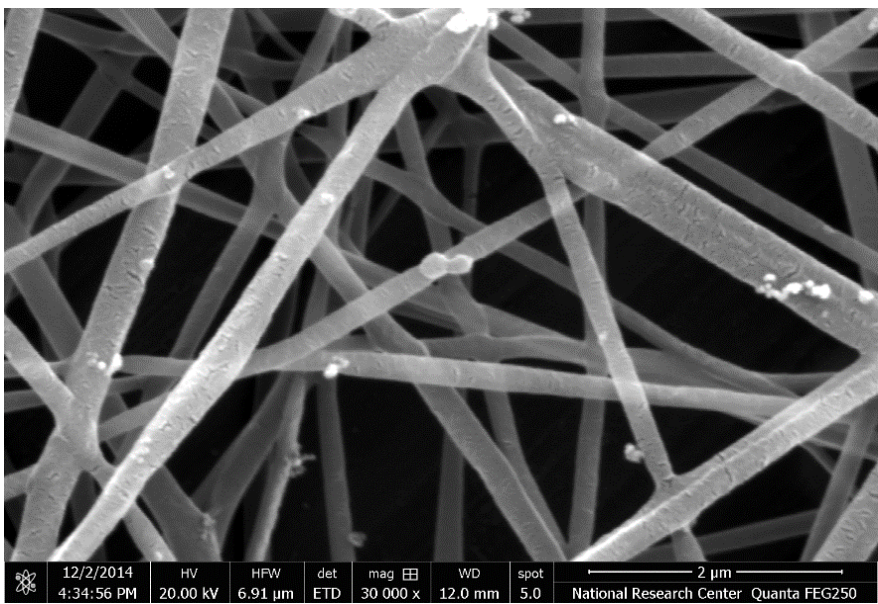

PVA/HEC loaded with 6\% (w/w) Drug

Fig. 4: SEM images for electro-spun fibers of PVA/HEC, PVA/HEC/2\% (w/w) Drug, PVA/HEC/4\% (w/w) Drug and PVA/HEC/6\% (w/w) Drug. Electrospinning parameters: applied voltage: $15 \mathrm{kV}$, flow rate: $2 \mathrm{ml} / \mathrm{h}$, gap distance: 25 $\mathrm{cm}$

\section{Differential scanning calorimetric}

DSC thermogram for the PVA/HEC and PVA/HEC/drug nanofibers with and without crosslinker are shown in Fig. 5 and Table 2. Drug proportion in samples was $6 \%(\mathrm{w} / \mathrm{w})$ of the total solid content. Polymers constituents comprise 70 parts of 10 $\%(\mathrm{w} / \mathrm{v})$ PVA and 30 parts of HEC 5\% (w/v).The physical properties of HEC are strongly dependent on the water absorbed because it is a highly hydrophilic polymer which typical for carbohydrate polymers (Hassabo et al., 2015). Therefore, there is a main endothermic peak, which attributed to the liberation of the absorbed moisture. On the other side, PVA has a sharp endothermic curve with a peak at $200^{\circ} \mathrm{C}$. At $190^{\circ} \mathrm{C}$, a melting endothermic peak was observed.

The endothermic curve for the PVA/HEC and PVA/HEC/drug nanofibers with or without crosslinker showed a peak that is shifted to the lower temperature. This movement confirms that, the crystalline microstructure of PVA/HEC is not as good as pure PVA nanofibers. In other words, the HEC reduces the crystallinity of the blend nanofibers. However, the presence of nicotinamide did not affect the thermal behaviour of the produced 
fiber. The endothermic peak of nanofibers exhibited a slight positive shifting in temperature with the addition of nicotinamide, which may be due to the segmental motions of polymer chains which formed by the interactions between them through hydrogen bonds.

One of the important physical parameter is the melting point that is used to identify the nature of the substance and its degree of purity which depends on the degree of crystallinity phase.

A single melting peak was observed at $215-221^{\circ} \mathrm{C}$ and $195-190^{\circ} \mathrm{C}$ before and after crosslinking, respectively. In Fig. 5, data showed that, the melting temperature for PVA/HEC and PVA/HEC/Drug nanofibers was similar, and it is nearly around the value of pure crystalline PVA, which is $200^{\circ} \mathrm{C}$.

Furthermore, the decrease in heat of fusion (listed in Table 2) in chemical crosslinking with citric acid suggested that the crystallinity are decreased. Crosslinking causes reduced hydration of the nanofibers that caused the higher temperature stability. The lower value of melting temperature after crosslinking due to the strong hydrogen bonds with the hydroxyl groups which indicate the compatibility between HEC and PVA.

It was also observed that the peak of melting temperature, from DSC curves, is decreased after crosslinking, which indicates that the nanofibers mat act like single network.

From DSC data, it is concluded that, the crosslinking of $\mathrm{PVA} / \mathrm{HEC} /$ cross and PVA/HEC/drug/cross nanofibers is thermally stable compared to PVA/HEC and PVA/HEC/drug nanofibers.

Table 2: DSC data and the characteristics observed for the produced nanofibers before and after crosslinking

\begin{tabular}{lll}
\hline Nanofibers & Melting temperature $\left({ }^{\circ} \mathrm{C}\right)$ & $\Delta \mathrm{H}(\mathrm{J} / \mathrm{g})$ \\
\hline PVA/HEC & 215 & 20 \\
PVA/HEC/crosslinker & 195 & 11 \\
PVA/HEC/Drug & 221 & 36 \\
PVA/HEC/Drug/crosslinker & 190 & 9 \\
\hline
\end{tabular}

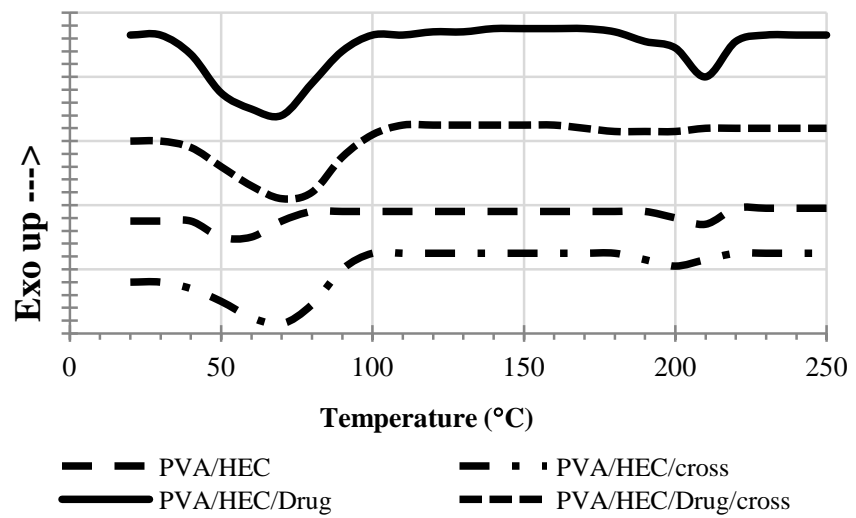

Fig. 5: DSC spectrum for PVA/HEC and PVA/HEC/Drug with and without citric acid.

\section{Thermal gravemetrical analysiss}

Thermal properties of PVA/HEC and PVA/HEC/drug nanofibers with and without cross-linker were measured and illustrated in Fig. 6 as TGA curves and their derivatives (dTGA).
The TGA curves exhibited three major weight losses during heating and they are more clearly in the diagram of derivative mass loss (dTGA \%). The first stage, (3 to $9 \%$ ) from 23 to $198^{\circ} \mathrm{C}$, affirms the presence of thermal process due to moisture evaporation or volatilization of small molecules from samples. Second weight loss, the major and the more significant stage (50 $77 \%$ ) in the range of $196-419^{\circ} \mathrm{C}$, includes the melting point and the degradation temperatures of polymers.

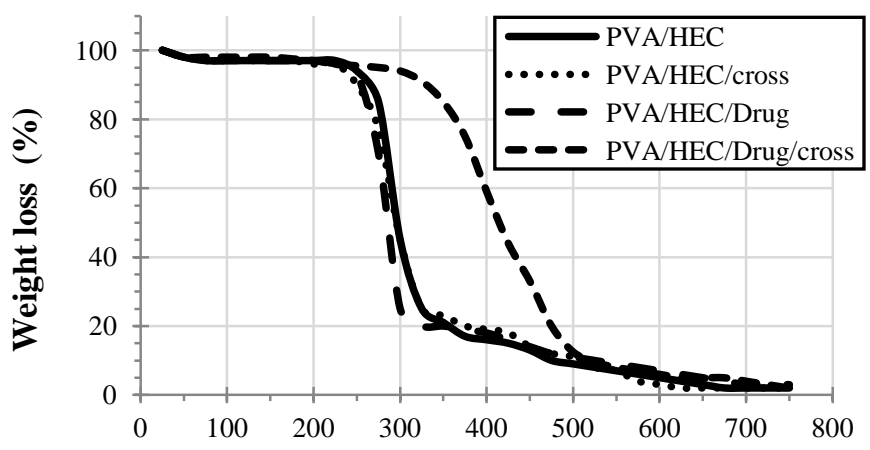

Temperature $\left({ }^{\circ} \mathrm{C}\right)$

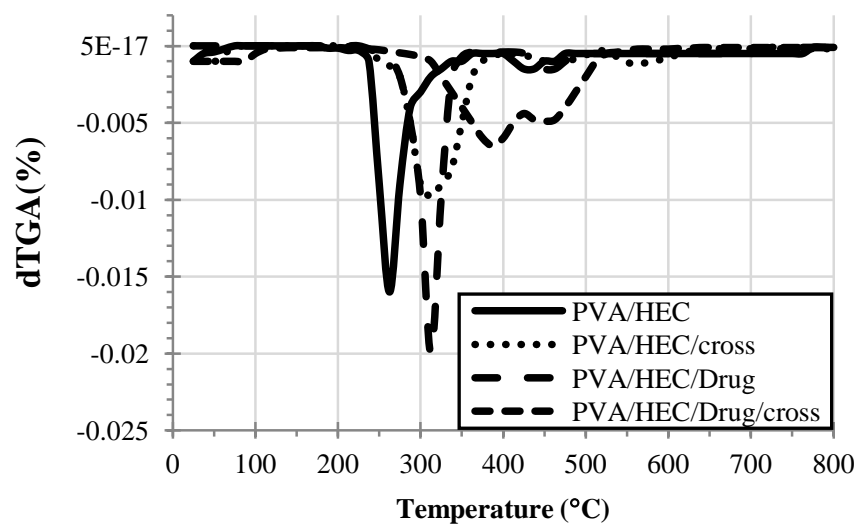

Fig. 6: TGA and DTGA for PVA/HEC and PVA/HEC/Drug with and without citric acid.

These values of weight loss proved the presence of chemical degradation process resulted from the polymeric backbones and referred to the degradation of PVA and HEC chains. The third weight loss at $350-530^{\circ} \mathrm{C}$ is due to the byproducts generated during the thermal degradation process. Furthermore, TGA data proved that, using crosslinker increases the thermal stability of produced nanofibers.

Thermal decomposition of PVA/HEC and PVA/HEC/drug nanofibers have different behaviour and it can be seen clearly from dTGA curves. DTGA curves show a corresponding peak temperature to the second and third decomposition regions. The maximum decomposition temperatures of PVA/HEC and PVA/HEC/drug nanofibers are increased in the presence of the chemical crosslinking (citric acid). In addition, PVA/HEC/Drug decomposition shifted to higher values and showed two decomposition temperature peaks. The 
latter phenomenon represents that citric acid has affected the thermal stability of the nanofibers by making a network between both the polymers. From TGA and dTGA results, it can be concluded that the thermal stability of produced nanofibers with drug increased when used citric acid as cross linker.

\section{Release profile and encapsulation efficiency for the drug}

The release characteristics of nicotinamide loaded into PVA/HEC electro-spun fibers were carried out by calculating the total amount of the drug released over time. Values were calculated using the calbration curve equation: $\mathrm{y}=0.0057 \mathrm{x}+$ 0.0186 and coefficient of determination $(\mathrm{R} 2)=0.9962$. Figure 7 shows the release profile of drug from the PVA/HEC/nicotinamide mats with and without citric acid. Samples with citric acid exposed to thermofixation at $150^{\circ} \mathrm{C}$ for 4 mints. $2 \%(w / w)$ concentration of nicotinamide was used for the study in which $50 \mathrm{mg}$ sample theoretically contains $1 \mathrm{mg}$ of nicotinamide. Figure 7 shows that samples with and without the citric acid released mostly $80 \%$ of the drug after $2 \mathrm{hrs}$ and $24 \mathrm{hrs}$ respectively. This rapid release profile for the uncorss-linked matrix is attributed to the affinity of both the matrix and the drug by the aqueous releasing medium. It was observed also that encapsulation efficiency are almost the same with and without crosslinking. This may be due to the entrapment space for drug between polymers chains did not show significant difference with and without crosslinking especially when low drug concentration used (Kurkuri and Aminabhavi, 2004). Nicotinamide release was controlled and extended over 24 hrs with crosslinking. Citric acid crosslinked PVA and HEC chains through esterification reaction described in Figure 2. Such crosslinking between chains resulting in a decrease in swelling of the water-soluble matrix of the two polymers PVA and HEC.

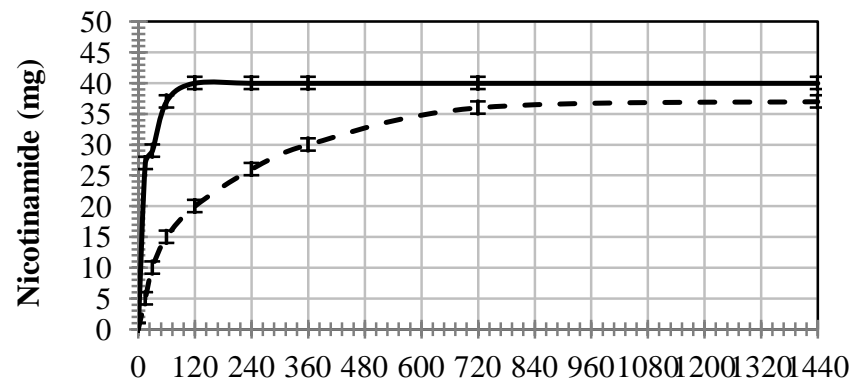

Time (min)

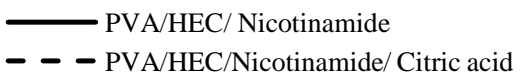

Fig. 7: Release profile of the PVA/HEC/nicotinamide with and without citric acid. Samples were incubated for $24 \mathrm{hrs}$ in PBS solution at $37^{\circ} \mathrm{C}$. Crosslinking condition: $5 \%(\mathrm{w} / \mathrm{w})$ citric acid and $50 \% \mathrm{w} / \mathrm{w}$ of citric acid were added to $\mathrm{PVA} / \mathrm{HEC} /$ Drug matrix, air-dried, and cured at $150^{\circ} \mathrm{C}$ for 4 mints.

\section{Cytotoxicity assessment}

Biocompatibility of the produced HEC/PVA/ nicotinamide electrospun mats was demonstrated by using immortalized human skin fibroblasts cells (hTERT-BJ1). Tested samples (Pure HEC, pure PVA, pure nicotinamide and
HEC/PVA/nicotinamide corsslinked with citric acid) were incubated in DMEM medium for 24 hours for extraction. Different concentrations $(12.5,25,50$ and $100 \mathrm{ppm})$ were tested for cytotoxicity. Only at $100 \mathrm{ppm}$ concentration of extracted medium showed detectable readings for cell cytotoxicity. Figure (8) shows the cell cytotoxicity of hTERT-BJ1cells which revealed that cell cytotoxicity was below $5 \%$. Samples of the crosslinked mat recoded the lowest value of cytotoxicity. Citric acid as a chemical crosslinker reduces the released residuals from samples (Van Luyn et al., 1995). The presented results have shown that HEC/PVA/Drug/Crosslinked mat and its constituents were proved to non-toxic matrix and recommended for medical purposes.

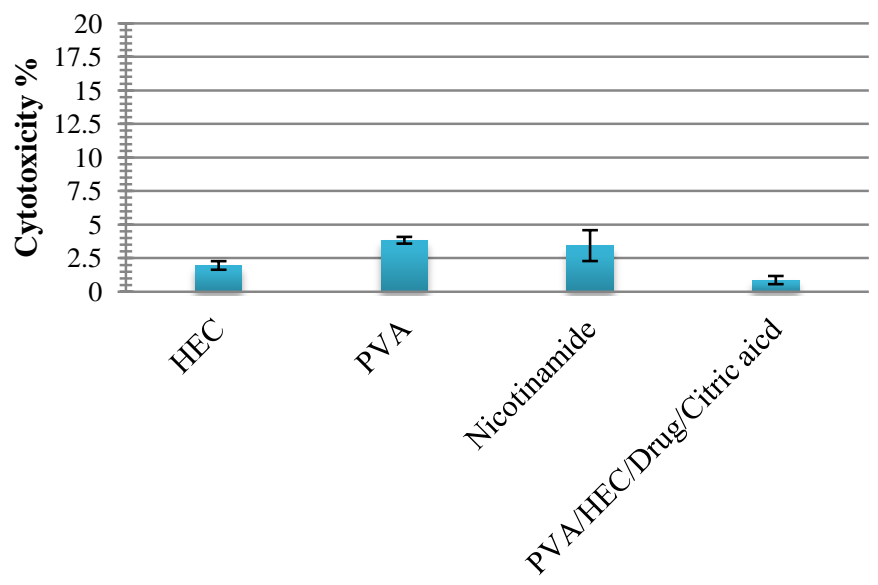

Tested Compounds

Fig. 8: Cell cytotoxicity of hTERT-BJ1 cells treated with electro-spun mat of HEC, PVA, nicotinamide and PVA/HEC/Drug/crosslinker. Tested samples were examined using $100 \mathrm{ppm}$ of the extracted medium.

\section{CONCLUSION}

$5 \%(w / v)$ of HEC mixed with $10 \%(w / v)$ of PVA in ratio $30: 70(\mathrm{v} / \mathrm{v})$ to form a spinnable water-soluble matrix was successfully electrospun. Electrospun parameters were adjusted to $20 \mathrm{kV}, 25 \mathrm{~cm}$ air gab and flow rate $2 \mathrm{ml} / \mathrm{h}$. Nicotinamide was successfully encapsulated in the HEC/PVA with different concentrations $(2,4,6 \% \mathrm{w} / \mathrm{w})$ without affecting the fiber morphology. SEM images showed uniform, porous, bead-free and nano-sized fibrous structures with diameter size 100-300 nm. FTIR indicated that there is interactions between HEC and PVA and proved the chemical reactions between $\mathrm{OH}$ groups of PVA and HEC with citric acid in the presence of sodium hypophosphite. The thermal analysis of the produced fibers showed that, the addition of HEC reduce the crystallinity of the HEC/PVA composite comparing with PVA itself, while, addition of drug did not affect the thermal behaviour of the fiber. Initial burst release of nicotinamide was recorded for uncrosslinked samples over the first two hours. However, nicrotinamide release was sustained over 24 hrs when PVA/HEC electrospun mat was crosslinked via $5 \%$ (w/w) of citric acid. Cytotoxicity of PVA/ HEC/ Drug/ Crosslinks against the immortalized human skin fibroblasts cells (hTERTBJ1) was very low. 


\section{ACKNOWLEDGEMENTS}

Authors are grateful to National Research Centre (Scopus affiliation ID: 60014618) for facilities provided. The authors have declared no conflicts of interest.

\section{REFERENCE}

Abdel-Azeem R., Nada A. Chitosan Liposomal microspheres for Ricinoleic acid Encapsulation. J. Appl. Pharm. Sci., 2015; 5(11), 055062.

Aravamudhan A., Ramos D. M., Nada A. A., Kumbar S. G. Natural Polymers: Polysaccharides and Their Derivatives for Biomedical Applications. In Natural and Synthetic Biomedical Polymers. Burlington, MA, USA: Elsevier: 67-89.

Charernsriwilaiwat N., Rojanarata T., Ngawhirunpat T., Sukma M., Opanasopit P. Electrospun chitosan-based nanofiber mats loaded with Garcinia mangostana extracts. Int. J. Pharm., 2013; 452(1-2), 333-43.

Cheng Y., Nada AA., Valmikinathan CM, Lee P., Liang D., Yu X., Kumbar S. G. In situ gelling polysaccharide-based hydrogel for cell and drug delivery in tissue engineering. J. Appl. Polym. Sci., 2014; 131(4), 39934.

Ghosh S. K. (Ed.). 2006. Functional Coatings: By Polymer Microencapsulation. Functional Coatings: By Polymer Microencapsulation. John Wiley \& Sons.

Hassabo A. G. New approaches to improving thermal regulating property of cellulosic fabric. Carbohydr. Polym., 2014; 101, 912-9.

Hassabo A. G., Nada A. A., Ibrahim H. M., Abou-Zeid N. Y. Impregnation of silver nanoparticles into polysaccharide substrates and their properties. Carbohydr. Polym., 2015; 122, 343-350.

Hassabo A. G., Salama M., Mohamed A. L., Popescu C. Ultrafine Wool and Cotton Powder and Their Characteristics. J. Nat. Fibers, 2015; 12(2), 141-153.

Huang X., Brazel C. S. On the importance and mechanisms of burst release in matrix-controlled drug delivery systems. J. Control. Release, 2001; 73(2-3), 121-136.

Hurst S. J. (Ed.). 2011. Biomedical Nanotechnology: Methods and Protocols. Illinois, USA: Humana Press.

Jin S. G., Kim K. S., Yousaf A. M., Kim D. W., Jang S. W., Son M.-W., Choi H.-G. Mechanical properties and in vivo healing evaluation of a novel Centella asiatica-loaded hydrocolloid wound dressing. Int. J. Pharm., 2015; 490(1-2), 240-247.

Kurkuri M. D., Aminabhavi T. M. Poly(vinyl alcohol) and poly(acrylic acid) sequential interpenetrating network $\mathrm{pH}$-sensitive microspheres for the delivery of diclofenac sodium to the intestine. J. Control. Release, 2004; 96(1), 9-20.

Lee K. Y., Jeong L., Kang Y. O., Lee S. J., Park W. H. Electrospinning of polysaccharides for regenerative medicine. Adv. Drug Deliv. Rev., 2009; 61(12), 1020-32.

Leyden J. J. Antibiotic resistant acne. Cutis, 1976; 17(3),593-6.

Nada A. A., Hassabo A. G., Fayad W., Awad H. M., Sleem A. A., Shaffie N. M., Zeid N. Y. A. Biomaterials Based on Essential Fatty Acids and Carbohydrates for Chronic Wounds. J. Appl. Pharm. Sci., 2015; 5(Suppl 3), 13-21.
Nada A. A., Hauser P., Hudson S. M. The grafting of per-(2,3,6O-allyl)- $\beta$ cyclodextrin onto derivatized cotton cellulose via thermal and atmospheric plasma techniques. Plasma Chem. Plasma Process., 2011; $31(4), 605-621$

Nada A. A., James R., Shelke N. B., Harmon M. D., Awad H. M., Nagarale R. K., Kumbar S. G. A smart methodology to fabricate electrospun chitosan nanofiber matrices for regenerative engineering applications. Polym. Adv. Technol., 2014; 25(5), 507-515.

Reddy N., Yang Y. Citric acid cross-linking of starch films. Food Chem., 2010; 118(3), 702-711.

Rezvanian M., Amin M.C.I.M., Ng S.-F. Development and physicochemical characterization of alginate composite film loaded with simvastatin as a potential wound dressing. Carbohydr. Polym., 2016; 137, 295-304.

Shalita AR., Smith J. G., Parish L. C., Sofman M. S., Chalker D. K. Topical nicotinamide compared with clindamycin gel in the treatment of inflammatory acne vulgaris. Int. J. Dermatol., 1995; 34(6), 434-437.

Skoog D. A., Holler F. J., Crouch S. R. 2007a. An Introduction to Infrared Spectrometry. In Principles of Instrumental Analysis (6th Editio, p. Chapt 16). Thomson Brooks/Cole: Chapt 16.

Skoog D. A., Holler F. J., Crouch S. R. (b). 2007b. Thermal Methods. In Principles of Instrumental Analysis (6th Editio, p. Chapt 31) Thomson Brooks/Cole: Chapt 31.

Supaphol P., Suwantong O. Electrospinning of biocompatible polymers and their potentials in biomedical applications. Biomed. Appl Polym. Nanofibers, 2012; 246, 213-239.

Taepaiboon P., Rungsardthong U., Supaphol P. Vitamin-loaded electrospun cellulose acetate nanofiber mats as transdermal and dermal therapeutic agents of vitamin A acid and vitamin E. Eur. J. Pharm. Biopharm., 2007; 67, 387-397.

Van Luyn M. J. A, Van Wachem N. B., Dijkstra P. J., Olde Damink L. H. H., Feijen J. Calcification of subcutaneously implanted collagens in relation to cytotoxicity, cellular interactions and crosslinking. J. Mater. Sci. Mater. Med., 1995; 6(5), 288-296.

Verreck G., Chun I., Peeters J., Rosenblatt J., Brewster M. E. Preparation and characterization of nanofibers containing amorphous drug dispersions generated by electrostatic spinning. Pharm. Res., 2003; 20(5), 810-817.

Xu X., Yang L., Xu X., Wang X., Chen X., Liang Q., Jing X. Ultrafine medicated fibers electrospun from W/O emulsions. J. Control. Release, 2005; 108(1), 33-42.

Yang Q., Li Z., Hong Y., Zhao Y., Qiu S., Wang C., Wei Y. Influence of solvents on the formation of ultrathin uniform poly(vinyl pyrrolidone) nanofibers with electrospinning. J. Polym. Sci. Part B Polym. Phys., 2004; 42(20), 3721-3726.

\section{How to cite this article:}

Nada AA, Hassabo AG, Mohamed AL, Zaghloul S. Encapsulation of Nicotinamide into Cellulose Based Electrospun Fibres. J App Pharm Sci, 2016; 6 (08): 013-021. 